BIOMEDICAL RESEARCH WITH CYCLOTRON PRODUCED RADIONUCLIDES, StEVEn M. Larson, M.D. PI.: Ronald D. Finn, Ph.D. Co-PI.

\title{
IMPROVING CANCER TREATMENT WITH CXCLOTRON PRODUCED RADIONUCLIDES
}

OVERALL OBJECTIVE: to promote nuclear medicine applications by improving the scientific basis for diagnosis, treatment and treatment follow-up with cyclotron produced tracers in oncology.

\section{Project 1. Cyclotron Innovations in target desigr and radiochemistry (R. Finn, PI).}

Objectives- Development of novel I-124, and Ga-66 targets, as well as methods of radiolabeling to antibodies, and production on "baby" cyclotrons; development of novel targets for radiolabeled precursors; radiolabeling of anti-tumor drugs, including FUDR, methotrexate, and the cross-resistant drug colchicine, for studies of multi-drug resistance in animal and human tumors. Novel enzymatic labeling methods first devised for $\mathrm{N}-13$ will be exploited to label with C-11, for thymidine and to a lesser extent, other metabolic tracers including $c-11$ amino acids.

Technical Progress Summary: The MSKCC cyclotron is now fully operational, with a new target changer(Japan steel Works) complete with targets of modified design (R. Finn, MSKCC), for the production of $\mathrm{F}-18$, and $\mathrm{N}-13$. Patient quality radiopharmaceuticals of [F-18] 2-fluoro-2-deoxy-(D)-glucose, 0-15-labeled water, and [F18]-5-fluoro-uridine have been prepared as described in the grant submission, and tested in animal models without problems. Initial characterization of the He-4 particle energies to produce Ga- $t 6$ using thin copper foil targets have been completed. Parameters for radiolabeling of I-124 have been determined which lead to retained immunoreactivity for the specific antibodies, $A-33$ and $3 F 8$, and these reagents have been used in basic animal studies using PET to quantify concentrations of antibody in tumors in vivo.

Articles published:

Gelbard AS, Cooper AJL, Asano Y, Nieves E, Filc-DeRicco S, Rosenspire KC: Methods for the enzymatic synthesis of tyrosine and phenylalanine labeled with nitrogen-13. Int $J$ Radiat Appl. Instr.Part A. 11:229-233, 1990.

Filc-DeRicco S, Gelbard AS, Cooper AJL, Rosenspire KC, Nieves E: Short-term metabolic fate of $\mathrm{L}-\left({ }^{15} \mathrm{~N}\right\}$ Glutamate in the walker 256 carcinosarcoma in vivo. Cancer Res 50:4839-4844, 1990.

Articles in Press:

Finn RD, Cheung NKV, Divgi C, st. Germaine J, Graham M, Pentlow $K$, Larson SM: Technical challenges associated with the radiolabeling of monoclonal antibodies utilizing short-lived positron emitting 
radionuclides. Int. J. Nucl. Med. Biol. 1990.

Finn RD, Knott $A$, Sheh $Y$, Tirelli $S$, Larson SM, Fissekis $J$, Laughlin JS: Rededication of the Memorial Sloan-Kettering Cancer Center Cyclotron. Nucl Instrum Methods. 1990.

Finn RD, Tirelli $S$, Sheh $Y$, Knott $A$, Gelbard AS, Larson SM: Consequences of electroplated taqrgets on radiopharmaceutical preparations. Nucl Instrum Methods. 1990.

Finn RD, Boothe TE: Quality Assurance considerations related to "in house" radiopharmaceutical preparations using positron emitting radionuclides. In A.Emran (ed) New Trends in Radiopharmaceutical Syntheses Quality control and Regulations. In Press.

Project 2. A novel method for assessing multi-drug resistance in vivo (J. Bading, PI; J. Biedler, J. O'Brien CO-PI's).

Objectives - Kinetic studies with long lived radiotracers $(\mathrm{H}-3$ and C-14) in multi-resistant and sensitive animal tumors of a variety of types will be pursued to develop an appropriate "crossresistant" drug that can serve as a marker substance for the demonstration of the multi-drug resistant phenotype in vivo. When the appropriate marker drug has been successfully radiolabeled with a positron emitter (our initial candidate is the drug colchicine), studies will be pursued in animals with PET imaging and if successful a demonstration project in humans is anticipated. Results of the radioactivity from the marker drug will be correlated with the quantitative expression of p-glycoprotein, using an unique antibody.

Technical progress Summary: MDR studies with sensitive (BE(2)C) and resistant $(B E(2)-C / C L C)$ variants of the human neuroblastoma cell line, have led to proof of principle studies using c-14 colchicine, and indicate that sensitive tumors retain significantly larger amounts of colchicine at time points up to an hour post injection, over a dose range varying from tracer to LD-50 doses of colchicine. Similar results have also been achieved with the melanoma tumor line $B E-2-C$, and $B E-2-C / C L C$ in the nude mouse. Also, there appears to be few metabolites of the radiolabeled colchicino circulating over the first hour. These studies suggest the possibility that colchicine retention by tumors can be used as a surrogate cross reactive marker for monitoring MDR expression by human tumors in vivo. Moreover, the precursor needed for ${ }^{11} \mathrm{C}-$ colchicine production has been synthesized.

Project 3 . Quantitative dosimetry based on positron labeled antitumor antibodies. (R. Pentlow, Project Leader).

Objectives- Positron emitting radionuclides with complex decay schemes, such as I-124 and $G a-66$, have not figured prominentily in applications to anti-tumor antibody dosinetry despite the obvious advantages of positron emission tomography for quantification. The reasons relate to concern about the ability to quantitatively 
image such radionuclides because of potential interference from time coordinated gamma rays that could fall in the time coilcident window at an energy which would allow them to be detectid as a coincident event. In a series of phantom studies, we have demonstrated that quantitative imaging of I-124 and $\mathrm{Ga-66}$ is possible under conditions likely to pertain in vivo, for "hotspot" imaging of tumors after parenteral injection. The purpose of this series of projects is to thoroughly evaluate the imaging physics of detection and quantification of I-124 and Ga-66 like radionuclides with modern PET scanners, and to extend the application to animal studies using a unique antibody system, radiolabeled $3 \mathrm{~F} 8$, an antibody against neuroblastoma tumor. These observations in animals would lay the foundation for quantitative dosimetry of radiolabeled anti-tumor antibodies. In addition, it is likely that animal studies would be pursued with other antibodies, such as $\mathrm{A}-33$, an anti-colon carcinoma antibody, and MX35, an anti-ovarian antibody. In addition, limited patient studies may be done (imaging and immunokinetic studies only).

Technical progress summary: In regard to (3), imaging physics studies have conclusively demonstrated for all of the diverse PET systems so far studied ( 6 distinct designs), that I-124 can be quantitatively imaged, even in the presence of large quantities, up to 100 times greater, of I-131. In two in-vivo animal tumor systems, namely, nude mice bearing neuroblastoma tumors, and nude rats bearing human ovarian cancer, the ability to measure concentrations of radiolabeled antibody in vivo has been validated. These studies have major implications for improved quantification of dosimetry for radiolabeled antibodies as weli as providing a basis for quantitative kinetic assessment of anti-tumor antibodies and their localization in human tumors in vivo.

Articles (in Press)

Pentlow KS, Graham MC, Lambrecht RM, Cheung NKV, Larson, SM: Quantitative imaging of $\mathrm{I}-124$ using positron emission tomography with applications to radioimmunodiagnosis and radioimmunotherapy

Medical Physics. 1990.

Abstracts Published:

Pentlow KS, Graham MC, Lambrecht RM, Larson SM. Quantitative imaging of radiolabelled antibodies Using Positron Emission Tomography. Medical Physics 16: 676, 1989.

Pentlow KS, Graham MC, Lambrecht RM, Larson, SM. Quantitative Imaging with Iodine-124 and positron Emission Tomography. Radiology 173 (P): 19l, 1989.

Kairemo KJA, Daghighian F., Brownell A-L, Rubin SC, Federici M., Pentlow KS, Larson SM. Positron emission tomography (PET) for diagnosis of ovarian cancer metastases using I-124 labeled monoclonal antibody in a nude rat model. J. Nucl. Med. 31:765, 1990 
Pentlow KS, Graham MC, Daghighian F, Finn R, Bacharach S, Bendriem $B$, Robeson, $W$. Lambrecht $R$, and Larson SM: The use of Positron emission tomography for quantitative imaging of I-124 Jabelled antibodies. J. Nucl. Med. 31: 864, 1990. 
, 